\title{
Molaren-Inzisiven-Hypomineralisation - Prävalenz, Ätiologie und Therapie
}

\author{
Molar-Incisor Hypomineralization - Prevalence, Etiology and \\ Therapy
}

Autor
Katrin Bekes

Institut

Fachbereich Kinderzahnheilkunde, Universitätszahnklinik, Medizinische Universität Wien, Österreich

\section{Schlüsselwörter \\ Molaren-Inzisiven-Hypomineralisation, MIH, Schmelz- defekte, Kinderzahnheilkunde}

Key words

molar-incisor hypomineralization, MIH, enamel defects, pediatric dentistry

Bibliografie

DOI https://doi.org/10.1055/a-0877-4633

Inf Orthod Kieferorthop 2019; 51: 141-146

(c) Georg Thieme Verlag KG Stuttgart · New York

ISSN 0020-0336

Korrespondenzadresse

Univ.-Prof. Dr. Katrin Bekes, MME

Fachbereich Kinderzahnheilkunde

Universitätszahnklinik

Medizinische Universität Wien

Sensengasse 2a1090 Wien

katrin.bekes@meduniwien.ac.at

\section{ZUSAMIMENFASSUNG}

Molaren-Inzisiven-Hypomineralisation (MIH) ist ein weltweit verbreitetes Problem. Die Ausprägung hypomineralisierter
Zahndefekte und die daraus resultierende Problematik können jedoch stark differieren. Zähne welche bereits kurz nach dem Durchbruch stark zerstört und dazu noch hypersensibel sind, erfordern andere Therapiepläne als nur leicht verfärbte, aber eigentlich gesunde Zähne. Eine frühzeitige Erkennung des Krankheitsbildes, die umfassende Betreuung und die Einleitung notwendiger therapeutischer Maßnahmen sind essenziell, um progressiven Schmelzverlusten entgegenzuwirken und einen langfristigen Zahnerhalt zu ermöglichen.

Der vorliegende Beitrag soll einen Einblick in die rezente Ätiologie, die Diagnostik und die therapeutischen Möglichkeiten der MIH geben und eine Hilfestellung für die Praxis sein.

\section{ABSTRACT}

Molar-incisor hypomineralization ( $\mathrm{MIH}$ ) is a worldwide problem. However, the severity of tooth defects caused by hypomineralization and the resulting problems can vary considerably. Teeth which are severely destroyed shortly after eruption and are hypersensitive require different treatment plans than only slightly discolored, but actually healthy teeth. Early recognition of the clinical picture, comprehensive care and the introduction of necessary therapeutic measures are essential in order to counteract progressive enamel loss and enable long-term tooth preservation.

This article is intended to give an insight into recent etiology, diagnostics and therapeutic possibilities of $\mathrm{MIH}$ and to provide practical assistance.

\section{Einleitung}

Während seit vielen Jahren in den industrialisierten Ländern ein rückläufiger Kariesbefall zu beobachten ist [1], beschäftigt ein anderes Phänomen mehr und mehr die Kinderzahnheilkunde: das gehäufte Auftreten einer speziellen Strukturstörung, der Molaren-Inzisiven-Hypomineralisation. Inwieweit Hypomineralisationen in der Vergangenheit durch den Kariesbefall überlagert wurden oder ob es sich um ein neues bzw. zunehmendes Phänomen handelt, konnte aus wissenschaftlicher Sicht bislang nicht geklärt werden. Cha- rakteristisch sind Schmelzfrakturen an frisch eruptierten, porös erscheinenden Molaren, eine Hypersensibilität der betroffenen Zähne und sich wiederholende Füllungsverluste [2].

\section{Definition und klinischer Befund}

Der Begriff Molaren-Inzisiven-Hypomineralisation beschreibt einen qualitativen Schmelzdefekt, bei welchem ein verringert mineralisierter, anorganischer Schmelzanteil zu Verfärbungen und Schmelz- 
abbrüchen bei den betroffenen Zähnen führen kann [3]. Die fehlstrukturierten Zähne zeichnen sich klinisch durch eine Veränderung in der Transluzenz des Schmelzes aus (> Abb. 1, 2). Geringgradig veränderte Zähne zeigen dabei eher weiß-gelbliche oder gelb-braune, unregelmäßige Verfärbungen, schwere Hypomineralisationsformen weisen dagegen abgesplitterte oder fehlende Schmelz- und/oder Dentinareale unterschiedlichen Ausmaßes auf [4]. Je dunkler die Farbe ist, umso weicher und poröser ist in der Regel der Zahnschmelz und umso höher ist damit die Gefahr des posteruptiven Substanzverlusts (meist an den Zahnhöckern) mit Dentinexposition [3]. Zur klinischen Einteilung des Schweregrads eignet sich die Klassifikation nach Wetzel und Reckel [5]:

- Grad I: Einzelne cremefarbene bis braune Areale an Kauflächen/ Höckerspitzen bei erhaltener Zahnmorphologie (\Abb. 3).

- Grad II: Überwiegend gelb-brauner Zahnschmelz, hypomineralisierte Bereiche über die Okklusalfläche hinaus bei erhöhter Gefahr für Schmelzfrakturen und gesteigerter Empfindlichkeit der betroffenen Zähne ( $\triangleright$ Abb. $\mathbf{3}$ ).

- Grad III: Große gelblich-braune Areale im gesamten Zahnbereich mit Defekten in der Kronenmorphologie und starken Schmelzverlusten gekoppelt mit einer hohen Empfindlichkeit der Zähne.

- Im Alltag zeigen sich die klinischen Manifestationen von MIH in den verschiedensten Bildern und Zuständen. Es können viele oder auch nur einzelne Zähne betroffen sein. Die Destruktionen können moderat, erheblich oder sehr gravierend sein. Dazu kommen mehr oder weniger willkürlich auftretende und unterschiedlich stark ausgeprägte Hypersensibilitäten insbesondere auf Kältereize, welche im Alltag zu einer natürlichen Schonhaltung führen können.

Ursprünglich wurde das Krankheitsbild für die ersten bleibenden Molaren und Inzisiven definiert. Dabei zeigte sich klinisch, dass die ersten Molaren meist häufiger und ausgeprägter befallen sind als die Schneidezähne [6]. Das klassische Bild dieses Befalls ist in der Zwischenzeit jedoch korrigiert worden, da vereinzelt als MIH beschriebene Defekte mittlerweile auch an anderen Zähnen der bleibenden Dentition (7er, 5er, 3er) beobachtet worden sind. Des Weiteren finden sich vermehrt auch Hypomineralisationen an den zweiten Milchmolaren und den Milcheckzähnen [7, 8].

\section{Prävalenz}

In Deutschland leiden etwa 4 bis 14 von 100 Kindern an MIH, in Österreich sind es wahrscheinlich im Durchschnitt 10,9\% [9-12]. Insgesamt tritt das Krankheitsbild weltweit auf, wobei die Angaben in der Literatur zur Prävalenz je nach Region schwanken. Berichtet wird von Häufigkeiten zwischen 2,8 und $44 \%$ [13]. Tendenziell scheint die MIH in Industrienationen häufiger als in anderen Ländern vorzukommen [14].

\section{Ätiologie}

Die Frage nach der möglichen Ursachen der MIH beschäftigt nicht nur die Eltern der betroffenen Kinder, sondern seit vielen Jahren auch die zahnmedizinische Forschung. Eine eindeutige Erklärung für das Phänomen gibt es allerdings bislang nicht, sodass die Ätio- logie des Krankheitsbildes bis dato immer noch als nicht geklärt betrachtet werden muss $[15,16]$. Als erschwerend in Bezug auf die Ursachenfindung ist insbesondere anzuführen, dass die Entwicklung der MIH bereits im Anfangsstadium der Zahnentwicklung mit einer Schädigung der Ameloblasten einhergeht und mit dem 4-6 Jahre späteren Durchbruch der bleibenden Zähne anamnestisch nur noch schwer korreliert werden kann.

Diskutiert wird ein multifaktorielles Geschehen. Als potentielle Ursachen werden in der Literatur angegeben: Probleme im letzten Monat der Schwangerschaft, Frühgeburt, häufige Erkrankungen in den ersten 4 Lebensjahren und verlängertes Trinken aus Plastiktrinkflaschen [17-19]. Weiterhin scheinen Durchfallerkrankungen, Fieberzustände und respiratorische oder bläschenbildende Erkrankungen evtl. eine Rolle zu spielen. Jüngst wurde Bisphenol A als ein ebenfalls möglicher Faktor genannt [20]. Darüberhinaus werden ätiopathogenetisch umwelttoxische Einflüsse in Erwägung gezogen [19].

\section{Diagnostik}

Diagnostische Kriterien für die MIH liegen von der EAPD aus dem Jahr 2003 vor [21]. Sie sind wie folgt festgelegt:

- klar umschriebene Opazitäten

- posteruptive Schmelzabbrüche

- atypische Restaurationen und Extraktionen

- atypische Hypersensibilitäten

Differenzialdiagnostisch müssen folgende Krankheitsbilder in Betracht gezogen werden: erbliche Strukturanomalien (Amelogenesis imperfecta), postnatale Entwicklungsstörungen (Dentalfluorose, Schmelzfehlbildungen durch Tetrazyklingabe) oder exogen bedingte Strukturanomalien (Trauma, apikale Entzündungen der Milchzähne (Turnerzahn), Karies).

\section{Therapie}

Eine Vermeidung der primären Ursachen von MIH ist aufgrund der noch immer mangelhaften Wissenslage bei der Ätiologie bis heute kaum möglich. Je nach Schweregrad der Hypomineralisation er-

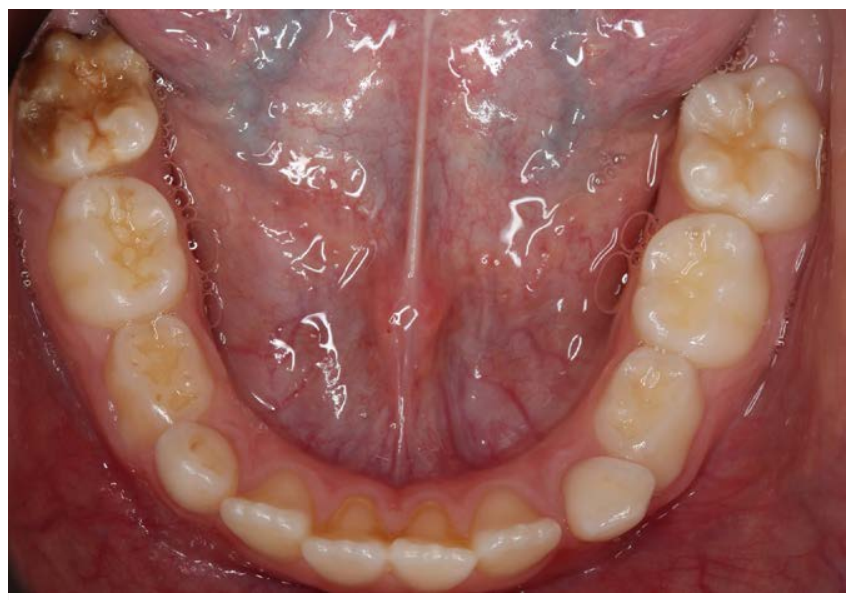

Abb. 1 MIH am Zahn 46 (Schweregrad 2). Zahn 36 ist gesund. 


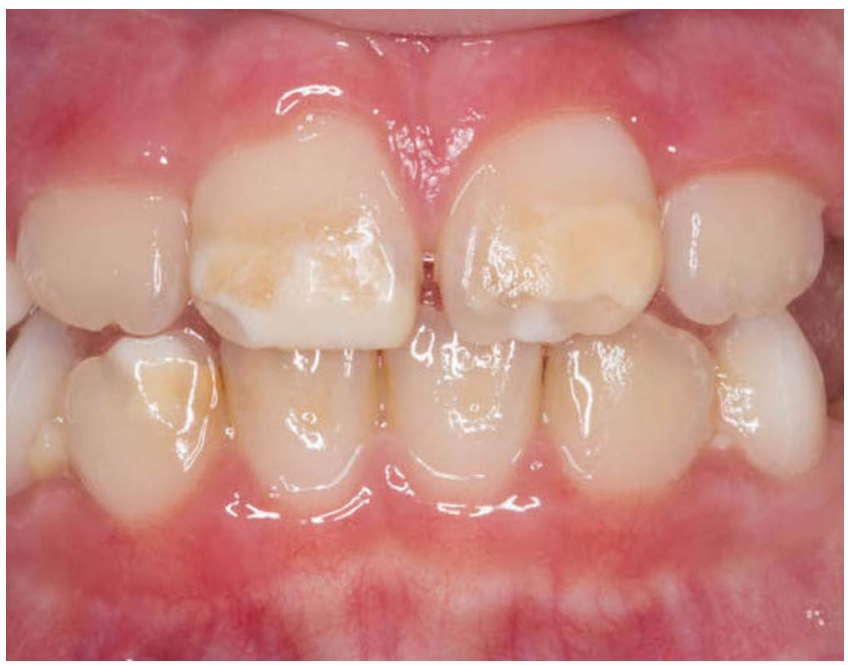

- Abb. 2 MIH an den Zähnen 11, 21 und 42.

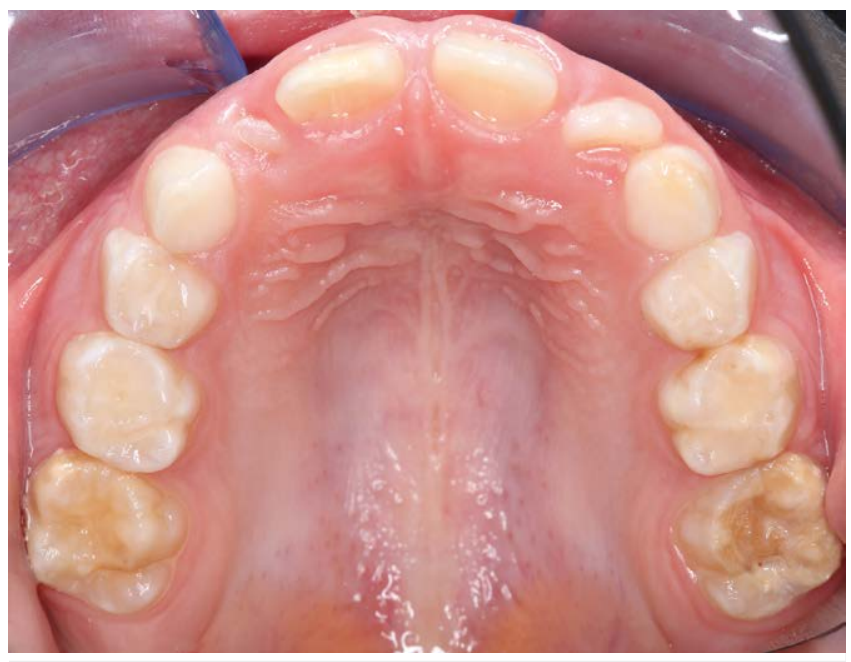

Abb. 3 MIH an den Zähnen 16 (Schweregrad 1) und 26 (Schwere$\operatorname{grad} 2)$.

streckt sich die zu favorisierende Therapie von der Intensivprophylaxe bis hin zu restaurativen Maßnahmen oder sogar der Extraktion. Diese zur Verfügung stehenden Möglichkeiten sind vielfach bekannt. Allerdings ergeben sich häufig bei den behandelnden Zahnärztinnen und Zahnärzten dahingehend Probleme, dass eine Unsicherheit bei der Wahl des „richtigen“ Therapiewegs besteht. Zur Entscheidungsfindung müssen verschiedene Faktoren gegeneinander abgewogen und eine für alle Beteiligten zufriedenstellende Lösungsstrategie gefunden werden.

Von MIH betroffene Frontzähne stellen meist aufgrund des geringeren Ausprägungsgrades der Hypomineralisation v. a. ein ästhetisches Problem dar [22]. Im Gegensatz dazu führt die MIH im Molarenbereich durch die mastikatorisch-funktionelle Belastung meist zu posteruptiven Schmelzabbrüchen mit konsekutiver Exposition des Dentins [2], insbesondere an den Höckerspitzen. Durch die raue und vergrößerte Oberfläche stellen diese Zähne auch Plaqueprädilektionsstellen dar [23], die zusammen mit der meist

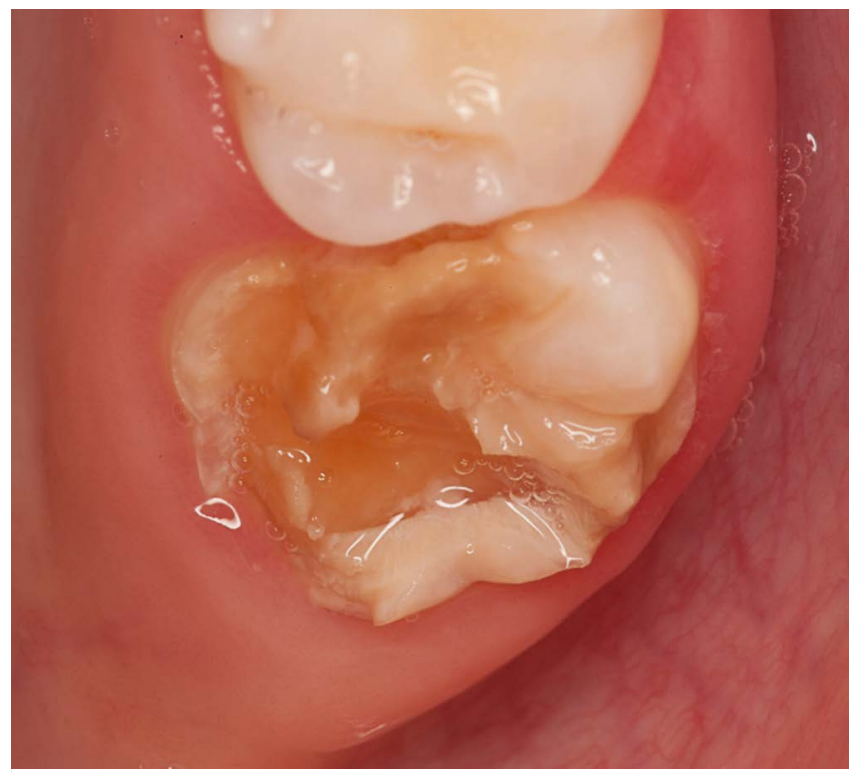

Abb. 4 Molaren-Inzisiven-Hypomineralisation Schweregrad 3.

schmerzbedingten reduzierten Mundhygiene eine erhöhte Kariesanfälligkeit zur Folge haben ( $\triangleright$ Abb. 4).

Unabhängig vom vorliegenden Schweregrad der MIH sollten alle betroffenen Kinder engmaschig in einem IntensivprophylaxeProgramm betreut werden. Hierzu werden folgende Maßnahmen empfohlen: regelmäßige Zahnreinigungen und Mundhygieneanleitungen, die Applikation von Fluorid-, CHX- und CPP-ACP-Präparaten [21], die Desensibilisierung mit einem Dentinadhäsiv oder einem Versiegler sowie die Applikation von Fissurenversieglungen. Fissurenversiegelungen können in leichten Fällen die Hypersensibilität verringern, in schweren Fällen aber den Hartsubstanzverlust nicht vorbeugen [22, 24].

Bei einer notwendigen restaurativen Therapie - sei es aufgrund von Karies oder einem posteruptiven Schmelzeinbruch - ist es häufig schwierig, im hypomineralisierten Schmelz eine exakte Ausdehnung der Restauration festzulegen und einzuschätzen, ob verfärbter jedoch noch nicht beschädigter MIH Schmelz nicht in naher Zukunft plötzlich frakturieren wird. Deshalb ist es nicht selten, dass es nach erfolgter Versorgung durchaus auch zu größeren Schmelzfrakturen am Rande der Restaurationen kommt. In der Literatur wird von einigen Autoren dennoch zunächst ein primär minimal invasives Vorgehen vorgeschlagen, verbunden mit periodischer Kontrolle der gelegten Füllung [25]. Als Füllungsmaterialien stehen dann verschiedene Möglichkeiten zur Auswahl, die von verschiedenen Faktoren abhängig sind, u. a. dem Alter der Patienten, der Ausdehnung und Qualität (Härte) der Zahnhartsubstanz, dem Schweregrad des Befalls, dem Ausmaß der Empfindlichkeit und der Lokalisation der betroffenen Zähne [22].

Für die Erstversorgung von Zähnen, bei denen aufgrund des noch unvollständigen Zahndurchbruchs keine absolute Trockenlegung möglich ist, eignen sich Glasionomerzemente, welche später durch definitive Füllmaterialien ausgetauscht werden sollten [24]. Molaren mit großen Substanzdefekten können zusätzlich proviso- 
risch mit einem schützenden Metallband (orthodontisches Band) provisorisch versorgt werden.

Im weiteren Verlauf bieten sich v. a. adhäsive Versorgungen mit Kompositen an [23] ( $\triangleright$ Abb. 5). Sie zeigen bei leichten bis mittleren Defekten bei adäquater Verarbeitung eine gute Überlebensrate auch bei hypomineralisierten Zähnen [26]. Allerdings muss aufgrund von Festigkeitsdefiziten von MIH befallenem Schmelz mit Absplitterungen am Füllungsrand gerechnet werden, was die Lebensdauer solcher Versorgungen reduziert. In In-Vitro-Untersuchungen zeigen dabei Self-Etch-Adhäsive bessere Haftwerte auf MIH geschädigten Zähnen als Total-Etch-Systeme [27]. Diese liegt allerdings noch immer unter der gewohnt hohen Schmelzhaftung gesunder Zähne.

Die Therapieoption bei größerem Substanzverlust und massiven Hypersensibilitäten zum Erhalt betroffener Molaren kann bei der Wahl einer Eingliederung einer konfektionierten Stahlkrone als Langzeitprovisorium liegen [28] ( $\mathbf{A b b}$. 6). Eine individuelle, prothetische Versorgung wird nicht bevorzugt, da neben einem hohen Kostenfaktor die jugendlichen Molaren eine große Pulpahöhle und nur kurze Kronen aufweisen. Dennoch ist im Gegensatz zum Gebrauch von konfektionierten Kronen bei Milchzähnen bei den ersten bleibenden Molaren auf eine möglichst schonende Präparation zu achten, damit genügend Substanz für die spätere definitive Ver-

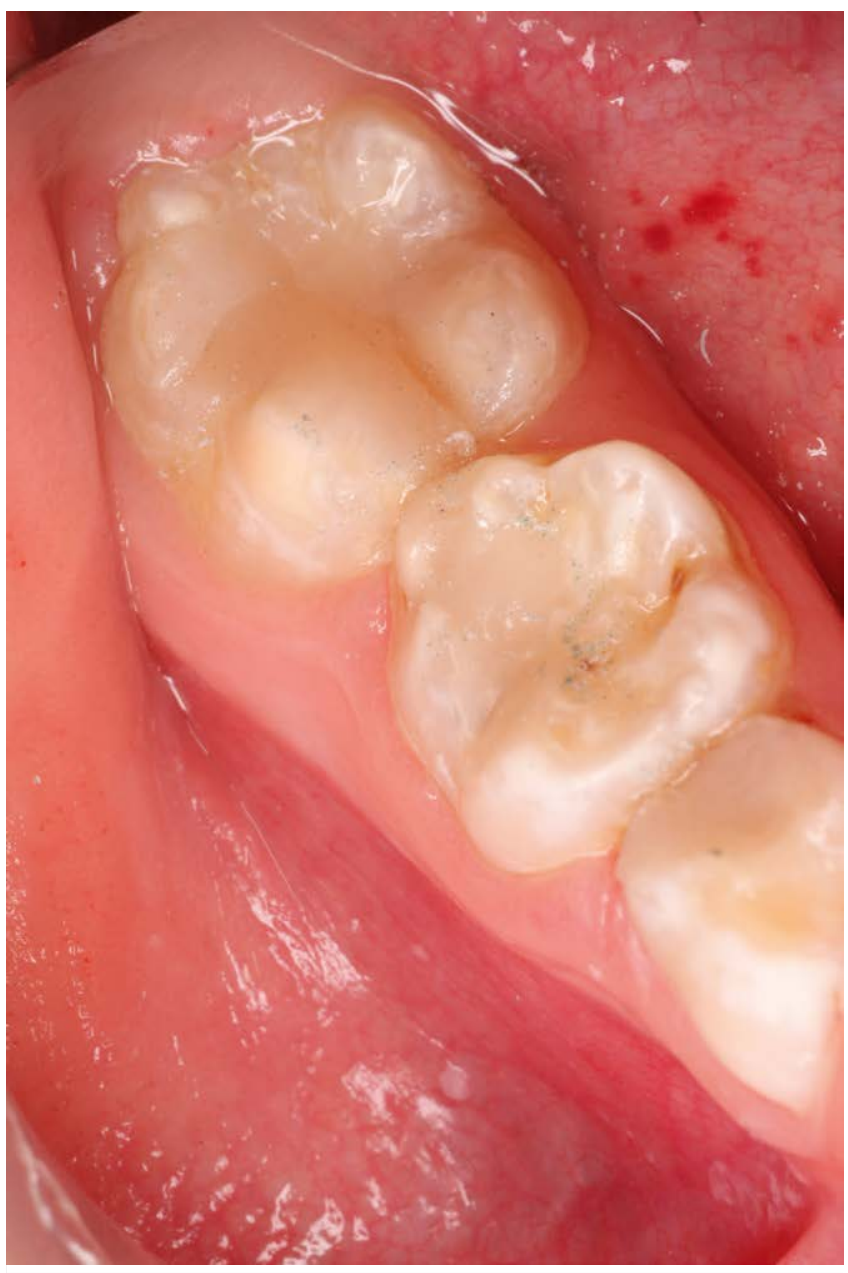

- Abb. 5 Kompostfüllung an einem MIH-Molaren. sorgung belassen wird. Nach dem 16. Lebensjahr kann die Stahlkrone dann durch eine Vollkeramik- oder VMK-Krone ersetzt werden.

Neuere Therapieansätze werben neben der Stahlkrone für den Einsatz laborgefertigter Arbeiten aus Komposit [29] ( $\mathbf{A b b}$. 7). Diese weisen den Vorteil auf, dass defektbezogen versorgt werden kann und es somit zu einem maximalen Schutz der nicht betroffenen Zahnhartsubstanz kommt.

Ist die Erhaltungswürdigkeit und Langzeitprognose des Molaren fraglich, stellt eine Extraktion mit nachfolgendem kieferorthopädischem Lückenschluss durch Mesialisation des zweiten und evtl. dritten Molaren in den meisten Fällen aufgrund des jugendlichen Alters des Patienten eine gute Therapieoption dar [30]. Diese Option kommt v. a. bei schweren Fällen mit extensivem Substanzverlust der ganzen Zahnkrone, starker kariöser Zerstörung und/oder endodontischen Problemen, einhergehend mit einem periapikalen pathologischen Prozess in Frage. Bei der Indikationsstellung und der zeitlichen Planung einer dieser Therapievarianten sollten der behandelnde Zahnarzt und der Kieferorthopäde eng interdiszipli-

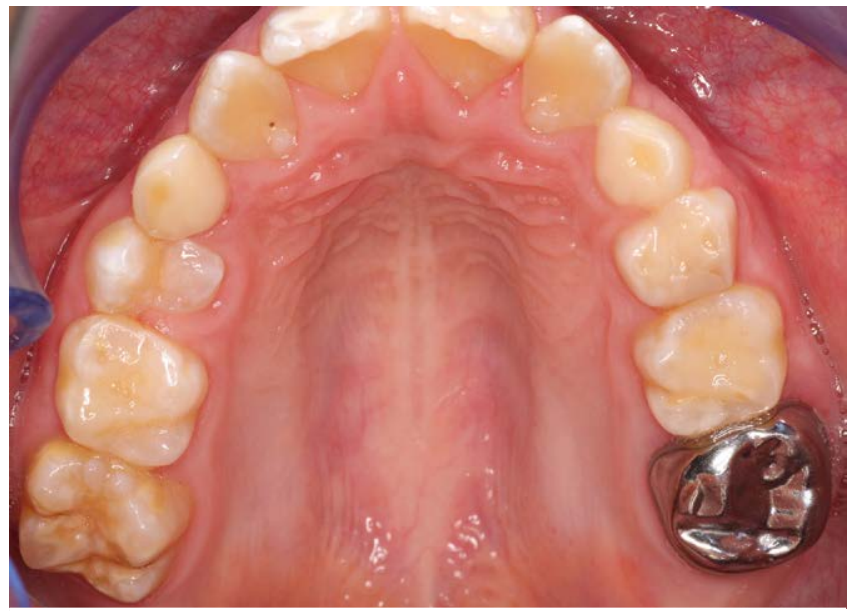

Abb. 6 Patient mit MIH an beiden oberen Molaren. Zahn 26 wurde bereits mit einer Stahlkrone versorgt.

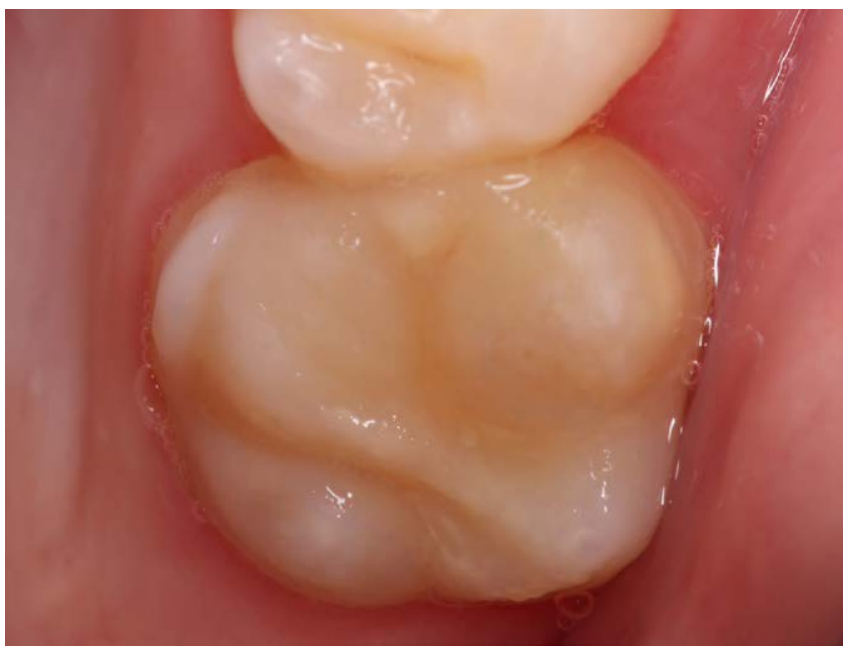

Abb. 7 Versorgung des MIH Molaren aus. 
när kooperieren, damit für den Patienten die bestmögliche Behandlungsstrategie bei möglichst kurzer Behandlungszeit erreicht werden kann [31].

Ergänzt werden sollte noch, dass die Behandlung vielfach zusätzlich durch die schlechte Anästhesierbarkeit der betroffenen hypomineralisierten Molaren erschwert wird [24]. In vielen Fällen bleibt die bestehende starke Hypersensibilität auch nach Verabreichung einer Lokalanästhesie weiterhin bestehen. Zur möglichen Lösung wird hierzu derzeit die Gabe von Schmerzmitteln (Paracetamol oder Ibuprofen) 24 h, 12 h, 6-8 h und direkt vor der eigentlichen Lokalanästhesie diskutiert [32].

\section{Zusammenfassung}

MIH ist ein weltweit verbreitetes Problem. Die Ausprägung hypomineralisierter Zahndefekte und die daraus resultierende Problematik können jedoch stark differieren. Zähne welche bereits kurz nach dem Durchbruch stark zerstört und dazu noch hypersensibel sind, erfordern andere Therapiepläne als nur leicht verfärbte, aber eigentlich gesunde Zähne. Eine frühzeitige Erkennung des Krankheitsbildes, die umfassende Betreuung und die Einleitung notwendiger therapeutische Maßnahmen sind essenziell, um progressiven Schmelzverlusten entgegenzuwirken und einen langfristigen Zahnerhalt zu ermöglichen.

\section{Erstveröffentlichung}

Dieser Beitrag wurde erstveröffentlicht in: Bekes K. Molaren-InzisivenHypomineralisation - Prävalenz, Ätiologie. ZWR - Das Deutsche Zahnärzteblatt 2017; 126: 32-36.

\section{Interessenkonflikt}

Es liegt kein Interessenkonflikt vor.

\section{Literatur}

[1] Jordan AR, Micheelis W. Fünfte Deutsche Mundgesundheitsstudie (DMS V). Köln: Deutscher Zahnärzte-Verlag 2016

[2] Weerheijm KL, Jalevik B, Alaluusua S. Molar-incisor hypomineralisation. Caries Res 2001; 35: 390-391

[3] Weerheijm KL, Duggal M, Mejare I et al. Judgement criteria for molar incisor hypomineralisation $(\mathrm{MIH})$ in epidemiologic studies: a summary of the European meeting on MIH held in Athens, 2003. Eur J Paediatr Dent 2003; 4: 110-113

[4] Koch G, Hallonsten AL, Ludvigsson N et al. Epidemiologic study of idiopathic enamel hypomineralization in permanent teeth of Swedish children. Community Dent Oral Epidemiol 1987; 15: 279-285

[5] Wetzel WE, Reckel U. Fehlstrukturierte Sechsjahrmolaren nehmen zu - eine Umfrage. Zahnärztl Mitt 1991; 81: 650-651

[6] Leppaniemi A, Lukinmaa PL, Alaluusua S. Nonfluoride hypomineralizations in the permanent first molars and their impact on the treatment need. Caries Res 2001; 35: 36-40
[7] Fuchs C, Buske G, Krämer N. Schmelzbildungsstörungen - Fallbericht einer generalisierten Schmelzbildungsstörung in der 1. Dentition (Enamel malformations - Case report of a generalised enamel malformation in the primary dentition). Oral Prophyl 2009; 31 : 178-186

[8] Elfrink ME, ten Cate JM, Jaddoe VW et al. Deciduous molar hypomineralization and molar incisor hypomineralization. J Dent Res 2012; 91: 551-555

[9] Dietrich G, Sperling S, Hetzer G. Molar incisor hypomineralisation in a group of children and adolescents living in Dresden (Germany). Eur J Paediatr Dent 2003; 4: 133-137

[10] Petrou MA, Giraki M, Bissar AR et al. Prevalence of Molar-Incisor-Hypomineralisation among school children in four German cities. Int J Paediatr Dent 2014; 24: 434-440

[11] Kuhnisch J, Heitmuller D, Thiering E et al. Proportion and extent of manifestation of molar-incisor-hypomineralizations according to different phenotypes. J Public Health Dent 2014; 74: 42-49

[12] Bürkle V. MIH - Hypomineralisation der Inzisiven und ersten bleibenden Molaren. Dentalhygiene. Journal 2008; 11: 20-22

[13] Elfrink ME, Ghanim A, Manton D] et al. Standardised studies on Molar Incisor Hypomineralisation (MIH) and Hypomineralised Second Primary Molars (HSPM): a need. Eur Arch Paediatr Dent 2015; 16: 247-255

[14] Steffen R, Krämer N, van Waes H. Molaren-Inzisiven-Hypomineralisation. Grundlagen, Ursachen, Präventionsansätze und Therapie. Zahnmedizin up2date 2015; 9: 313-324

[15] Crombie F, Manton D, Kilpatrick N. Aetiology of molarincisor hypomineralization: a critical review. Int J Paediatr Dent 2009; 19: 73-83

[16] Silva MJ, Scurrah KJ, Craig JM et al. Etiology of molar incisor hypomineralization - A systematic review. Community Dent Oral Epidemiol 2016; 44: 342-353

[17] Jalevik B, Noren JG, Klingberg $G$ et al. Etiologic factors influencing the prevalence of demarcated opacities in permanent first molars in a group of Swedish children. Eur J Oral Sci 2001; 109: 230-234

[18] Beentjes VE, Weerheijm KL, Groen HJ. Factors involved in the aetiology of molar-incisor hypomineralisation (MIH). Eur J Paediatr Dent 2002; 3: 9-13

[19] Souza JF, Costa-Silva CM, Jeremias F et al. Molar incisor hypomineralisation: possible aetiological factors in children from urban and rural areas. Eur Arch Paediatr Dent 2012; 13: 164-170

[20] Jedeon K, De la Dure-Molla M, Brookes S] et al. Enamel defects reflect perinatal exposure to bisphenol A. Am J Pathol 2013; 183: 108-118

[21] Lygidakis NA, Wong F, Jalevik B et al. Best Clinical Practice Guidance for clinicians dealing with children presenting with Molar-Incisor-Hypomineralisation (MIH): An EAPD Policy Document. Eur Arch Paediatr Dent 2010; 11: 75-81

[22] Fayle SA. Molar incisor hypomineralisation: restorative management. Eur J Paediatr Dent 2003; 4: 121-126

[23] Knapp V, Nies S. Molar-Incisor-Hypomineralization. Zahnmedizin up2date 2009; 3: 491-510

[24] Jalevik B, Klingberg GA. Dental treatment, dental fear and behaviour management problems in children with severe enamel hypomineralization of their permanent first molars. Int J Paediatr Dent 2002; 12: 24-32

[25] Kellerhoff NM, Lussi A. Molar-incisor hypomineralization. Schweiz Monatsschr Zahnmed 2004; 114: 243-253

[26] Lygidakis NA, Chaliasou A, Siounas G. Evaluation of composite restorations in hypomineralised permanent molars: a four year clinical study. Eur J Paediatr Dent 2003; 4: 143-148 
[27] William V, Messer LB, Burrow MF. Molar incisor hypomineralization: review and recommendations for clinical management. Pediatr Dent 2006; 28: 224-232

[28] Zagdwon AM, Toumba KJ, Curzon ME. The prevalence of developmental enamel defects in permanent molars in a group of English school children. Eur J Paediatr Dent 2002; 3: 91-96

[29] Feierabend S. Behandlungskonzepte bei Strukturanomalien des Zahnschmelzes und des Dentins. Stomatologie 2014; 111: 341-353
[30] Jalevik B, Moller M. Evaluation of spontaneous space closure and development of permanent dentition after extraction of hypomineralized permanent first molars. Int J Paediatr Dent 2007; 17: 328-335

[31] Williams JK, Gowans AJ. Hypomineralised first permanent molars and the orthodontist. Eur J Paediatr Dent 2003; 4: 129-132

[32] Steffen R, van Waes H. Die Behandlung von Kindern mit Molaren-Inzisiven-Hypomineralisation. Eine Herausforderung bei der Schmerzkontrolle und Verhaltenssteuerung. Quintessenz 2011; 62: 1585-1592 